\title{
Real photon emissions in leptonic decays
}

\section{G. Martinelli*i, M. Di Carlo, F. Mazzetti}

Physics Department and INFN Sezione di Roma La Sapienza, Piazzale Aldo Moro 5, 00185

Roma, Italy

\section{G.M. de Divitiis, A. Desiderio, R. Frezzotti, M. Garofalo, M. Hansen, N. Tantalo}

University of Rome Tor Vergata and INFN Roma Tor Vergata, Via della Ricerca Scientifica 1, I-00133, Rome, Italy

\section{D.Giusti, V. Lubicz}

Dip. di Matematica e Fisica, University of Roma Tre and INFN Roma Tre,

Via della Vasca Navale 84, I-00146 Rome, Italy

\section{C.T. Sachrajda}

Department of Physics and Astronomy, University of Southampton,

Southampton SO17 1BJ, UK

\section{F. Sanfilippo, S. Simula}

INFN Roma Tre,

Via della Vasca Navale 84, I-00146 Rome, Italy

\begin{abstract}
We present a non-perturbative calculation of the form factors which contribute to the amplitudes for the radiative decays $P \rightarrow \ell \bar{v}_{\ell} \gamma$, where $P$ is a pseudoscalar meson and $\ell$ is a charged lepton. Together with the non-perturbative determination of the virtual photon corrections to the processes $P \rightarrow \ell \bar{v}_{\ell}$, this will allow accurate predictions to be made at $O\left(\alpha_{e m}\right)$ for leptonic decay rates for pseudoscalar mesons ranging from the pion to the $B$ meson. We are able to separate unambiguously the point-like contribution, the square of which leads to the infrared divergence in the decay rate, from the structure dependent, infrared-safe, terms in the amplitude. The fully non-perturbative, $O(a)$ improved calculation of the inclusive leptonic decay rates will lead to significantly improved precision in the determination of the corresponding Cabibbo-KobayashiMaskawa (CKM) matrix elements. Precise predictions for the emission of a hard photon are also very interesting, especially for the decays of heavy $D$ and $B$ mesons for which currently only model-dependent predictions are available to compare with existing experimental data.
\end{abstract}

37th International Symposium on Lattice Field Theory - Lattice2019

16-22 June 2019

Wuhan, China

* Speaker.

†guido.martinelli@roma1.infn.it 


\section{Introduction}

The determination of the CKM matrix elements represents a crucial test of the limits of the Standard Model (SM) in the quest for new physics. In this framework, precise experimental measurements and accurate theoretical predictions of the leptonic decay rates of light and heavy pseudoscalar mesons are particularly important. A first-principles calculation of these quantities requires the non-perturbative determination of the physical amplitudes/rates that can only be obtained from QCD simulations on the lattice. In order to fully exploit the available experimental information [1], strong isospin-breaking effects and $O\left(\alpha_{e m}\right)$ electromagnetic corrections must be included. In particular, we must be able to compute the rates for radiative leptonic decays $P \rightarrow \ell \bar{v}_{\ell} \gamma$, where $P$ is a charged pseudoscalar meson, $\gamma$ a photon, $\ell$ a (anti-)lepton and $\bar{v}_{\ell}$ the corresponding anti-neutrino (neutrino). This would also allow accurate, model-independent predictions of the important radiative decays of heavy mesons with the emission of a hard photon. Results of a lattice calculation of real-photon emission amplitudes have also been presented at this conference in ref. [2].

In the limit of soft-photon energies, the radiative decay rate can be reliably calculated perturbatively by treating the meson as a point-like particle. This limit is however an idealisation and experimental measurements are inclusive up to photon energies that might be too large to neglect structuredependent (SD) corrections to the point-like approximation. The region of hard photon energies, which is particularly important for heavy mesons, represents a fundamental probe of the internal structure of the mesons and can only be studied in lattice QCD simulations. On the other hand, even in the case of light mesons, where chiral perturbation theory can be used, the low-energy constants entering at $O\left(p^{6}\right)$ can only be estimated using model-dependent assumptions [3]-[7].

In ref. [8] a strategy to compute QED radiative corrections to the $P \rightarrow \ell \bar{v}_{\ell}(\gamma)$ decay rates at $O\left(\alpha_{e m}\right)$ on the lattice was proposed. The strategy was subsequently applied to provide the first non-perturbative model-independent calculation of the decay rates $\pi^{-} \rightarrow \mu^{-} \bar{v}_{\mu}(\gamma)$ and $K^{-} \rightarrow$ $\mu^{-} \bar{v}_{\mu}(\gamma)$ [9]-[13]. The real soft-photon contributions was calculated in the point-like effective theory and the SD corrections were estimated, by relying on the quoted chiral perturbation theory results, to be negligible (see [8]). On the other hand SD corrections might be relevant for the decays of pions and kaons into electrons when the energy of the photon becomes larger than about $20 \mathrm{MeV}$. Moreover, in the single-pole dominance approximation proposed in ref. [14], the SD contribution was estimated to be rather large in the case of heavy flavours. This contribution can be precisely determined only in lattice QCD. Here we present a non-perturbative, $O(a)$ improved lattice calculation of the form factors entering the radiative decay rate $P \rightarrow \ell \bar{v}_{\ell} \gamma$ in the case of pions, kaons, $D$ and $D_{s}$ mesons. The case of bottom mesons will be studied in a future work on the subject.

\section{Form factors contributing to the radiative decay amplitude}

The non-perturbative hadronic amplitude for the process $P \rightarrow \ell v_{\ell} \gamma$ is given by the T-product

$$
H_{W}^{\alpha r}(k, p)=\varepsilon_{\mu}^{r}(k) H_{W}^{\alpha \mu}(k, p)=\varepsilon_{\mu}^{r}(k) \int d^{4} y e^{i k \cdot y}\left\langle 0\left|\mathrm{~T}\left\{j_{W}^{\alpha}(0) j_{e m}^{\mu}(y)\right\}\right| P(\boldsymbol{p})\right\rangle,
$$


where $\varepsilon_{\mu}^{r}(k)$ is the polarization vector of the photon with four-momentum $k, j_{e m}^{\mu}$ is the electromagnetic current, $j_{W}^{\alpha}$ is the hadronic weak current, $j_{W}^{\alpha}=V^{\alpha}-A^{\alpha}=\bar{q}_{1}\left(\gamma^{\alpha}-\gamma^{\alpha} \gamma_{5}\right) q_{2}$, and $\boldsymbol{p}$ is the momentum of the meson $P$ with mass $m_{P}$. To this amplitude, at $O\left(\alpha_{e m}\right)$, we have to add the diagram in which the photon is emitted from the final-state charged lepton. The latter contribution can however, be computed in perturbation theory using the meson decay constant $f_{P}$. The decomposition of $H_{W}^{\alpha r}(k, p)$ in terms of form-factors has been discussed, for example, in refs. [8, 15]

$$
\begin{aligned}
& H_{W}^{\alpha r}(k, p)=\varepsilon_{\mu}^{r}(k)\left\{H_{1}\left[k^{2} g^{\mu \alpha}-k^{\mu} k^{\alpha}\right]+H_{2}\left[\left(p \cdot k-k^{2}\right) k^{\mu}-k^{2}(p-k)^{\mu}\right](p-k)^{\alpha}\right. \\
& \left.-i \frac{F_{V}}{m_{P}} \varepsilon^{\mu \alpha \gamma \beta} k_{\gamma} p_{\beta}+\frac{F_{A}}{m_{P}}\left[\left(p \cdot k-k^{2}\right) g^{\mu \alpha}-(p-k)^{\mu} k^{\alpha}\right]+f_{P}\left[g^{\mu \alpha}+\frac{(2 p-k)^{\mu}(p-k)^{\alpha}}{2 p \cdot k-k^{2}}\right]\right\} .
\end{aligned}
$$

The last term in Eq. (2.2) corresponds to the point-like infrared-divergent contribution. This term saturates the Ward Identity satisfied by $H_{W}^{\alpha \mu}(k, p)$, i.e. $k_{\mu} H_{W}^{\alpha \mu}(k, p)=i\left\langle 0\left|j_{W}^{\alpha}(0)\right| P(p)\right\rangle=f_{P} p^{\alpha}$. The four form-factors $H_{1,2}$ and $F_{V, A}$ are scalar functions of Lorentz invariants, the squared meson mass $m_{P}^{2}, p \cdot k$ and $k^{2}$. Eq. (2.2) is valid for generic (off-shell) values of the photon momentum and for generic choices of the polarisation vectors. By setting the photon on-shell, i.e. by taking $k^{2}=0$, at fixed meson mass the form factors are functions of $p \cdot k$ only. A convenient dimensionless variable is given by $x_{\gamma}=2 p \cdot k / m_{P}^{2}$. By choosing a physical basis for the polarization vectors such that $\varepsilon_{r} \cdot k=0$ we have

$$
H_{W}^{\alpha r}(k, p)=\varepsilon_{\mu}^{r}(k)\left\{-i \frac{F_{V}\left(x_{\gamma}\right)}{m_{P}} \varepsilon^{\mu \alpha \gamma \beta} k_{\gamma} p_{\beta}+\left[\frac{F_{A}\left(x_{\gamma}\right)}{m_{P}}+\frac{f_{P}}{p \cdot k}\right]\left(p \cdot k g^{\mu \alpha}-p^{\mu} k^{\alpha}\right)+\frac{f_{P}}{p \cdot k} p^{\mu} p^{\alpha}\right\} .
$$

Once the decay constant $f_{P}$ and the two SD axial and vector form-factors $F_{A}$ and $F_{V}$ are known, the decay rate can be calculated by using the formulae given in [15] and in appendix B of [8].

\section{Extracting the form factors from Euclidean correlators}

The Euclidean correlation function corresponding to Eq. (2.1) is given by

$$
C_{W}^{\alpha r}(t, \boldsymbol{p}, \boldsymbol{k})=-i \varepsilon_{\mu}^{r}(\boldsymbol{k}) \int d^{4} y \int d^{3} \boldsymbol{x}\left\langle 0\left|\mathrm{~T}\left\{j_{W}^{\alpha}(t, \mathbf{0}) j_{e m}^{\mu}(y)\right\} P(0, \boldsymbol{x})\right| 0\right\rangle e^{E_{\gamma} t_{y}-i \boldsymbol{k} \cdot \boldsymbol{y}+i \boldsymbol{p} \cdot \boldsymbol{x}}
$$

where $k=\left(i E_{\gamma}, \boldsymbol{k}\right)$, with $E_{\gamma}=|\boldsymbol{k}|, p=(i E, \boldsymbol{p})$ and $\int d^{3} \boldsymbol{x} P(0, \boldsymbol{x}) e^{i \boldsymbol{p} \cdot \boldsymbol{x}}$ is the source of the pseudoscalar meson with momentum $\boldsymbol{p}$. The convergence of the integral over $t_{y}$ is ensured by the safe analytic continuation from Minkowski to Euclidean space, because of the absence of intermediate states lighter than the pseudoscalar meson. The physical form factors can be extracted directly from the Euclidean correlation functions

$$
R_{W}^{\alpha r}(t ; \boldsymbol{p}, \boldsymbol{k})=\frac{2 E}{e^{-t\left(E-E_{\gamma}\right)}\langle P(\boldsymbol{p})|P| 0\rangle} C_{W}^{\alpha r}(t ; \boldsymbol{p}, \boldsymbol{k})=H_{W}^{\alpha r}(k, p)+\cdots
$$

where $\langle P(\boldsymbol{p})|P| 0\rangle$ is the matrix element of the operator $P$ between the vacuum and the meson state and the dots represent sub-leading exponentials. It is useful to note that, in order to separate the 

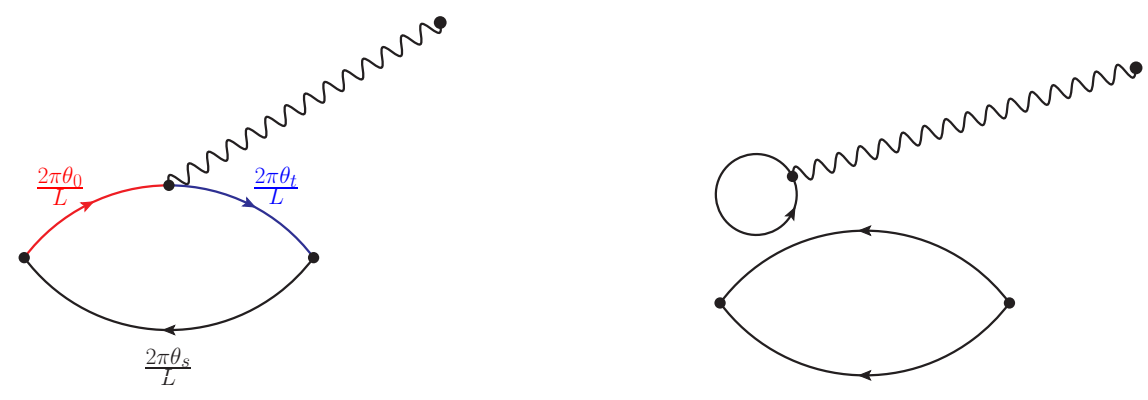

Figure 1: The connected diagram on the left shows our choice of the spatial boundary conditions. By treating the two propagators attached to the electromagnetic current as two different flavours, with the same mass and electric charge but different boundary conditions, we may choose arbitrary values for the meson and photon spatial momenta.The diagram on the right represents the contribution associated with the emission of the photon by the sea-quarks. By neglecting this diagram we have been working in the so-called electro-quenched approximation.

axial and vector form-factors it is enough to compute separately the ratios $R_{V, A}^{\alpha r}(t ; \boldsymbol{p}, \boldsymbol{k})$ corresponding to the (renormalised) vector and axial component of the weak current, see eq. (3.4) below. For $j_{e m}^{\mu}$ an exactly conserved lattice vector current is employed. The previous discussion assumed an infinite time extent $(T)$ of the lattice. In our numerical calculations we have employed numerical estimators for the ratios $R_{V, A}^{\alpha r}(t ; \boldsymbol{p}, \boldsymbol{k})$ built in terms of finite- $T$ correlators that properly account for the fact that the simulated quark and gauge fields satisfy respectively anti-periodic and periodic boundary conditions in time.

Within the electro-quenched approximation, i.e. in the absence of the disconnected contribution shown in the right-panel of Fig. 1, it is possible to choose arbitrary values of the spatial momenta by using different spatial boundary conditions $[16,17]$ for the quark fields. More precisely, we set the boundary conditions for the "spectactor" quark such that $\psi(x+\hat{\boldsymbol{k}} L)=\exp \left(2 \pi i \hat{\boldsymbol{k}} \cdot \boldsymbol{\theta}_{s} / L\right) \psi(x)$. Then we treat the two propagators that are connected with the electromagnetic current (the red and blue lines) as the results of the Wick contractions of two different fields having the same mass and electric charge but satisfying different boundary conditions. This is possible at the price of accepting tiny violations of unitarity that are exponentially suppressed in the volume (similar effects are induced in any case by the electro-quenched approximation). By setting the boundary conditions as illustrated in the figure, we have thus been able to choose arbitrary values for the meson and photon spatial momenta, $\boldsymbol{p}=\frac{2 \pi}{L}\left(\boldsymbol{\theta}_{0}-\boldsymbol{\theta}_{s}\right)$ and $\boldsymbol{k}=\frac{2 \pi}{L}\left(\boldsymbol{\theta}_{0}-\boldsymbol{\theta}_{t}\right)$ by tuning the real three-vectors $\boldsymbol{\theta}_{0, t, s}$, where the subscript $i=0, t, s$ in the definition refers to the quark line emerging from the source in the origin, 0 ; the quark annihilating in the sink given by the hadronic weak current at time $t$ and the spectator quark respectively. The numerical results have been obtained by choosing all the non-zero components of the spatial momenta to be along the z-direction, i.e. $\boldsymbol{p}=(0,0,|\boldsymbol{p}|)$ and $\boldsymbol{k}=\left(0,0, E_{\gamma}\right)$. With this particular choice a convenient basis for the polarization vectors of the photon is the one in which the two physical polarization vectors are given by $\varepsilon_{1,2}^{\mu}=$ $\left(0, \mp \frac{1}{\sqrt{2}},-\frac{1}{\sqrt{2}}, 0\right)$. In this basis we have $\varepsilon_{r} \cdot p=\varepsilon_{r} \cdot k=0$ and consequently

$$
H_{A}^{j r}(k, p)=\frac{\varepsilon_{r}^{j} m_{P}}{2} x_{\gamma}\left[F_{A}\left(x_{\gamma}\right)+\frac{2 f_{P}}{m_{P} x_{\gamma}}\right], \quad H_{V}^{j r}(k, p)=\frac{i\left(E_{\gamma} \boldsymbol{\varepsilon}_{r} \wedge \boldsymbol{p}-E \boldsymbol{\varepsilon}_{r} \wedge \boldsymbol{k}\right)^{j}}{m_{P}} F_{V}\left(x_{\gamma}\right) .
$$



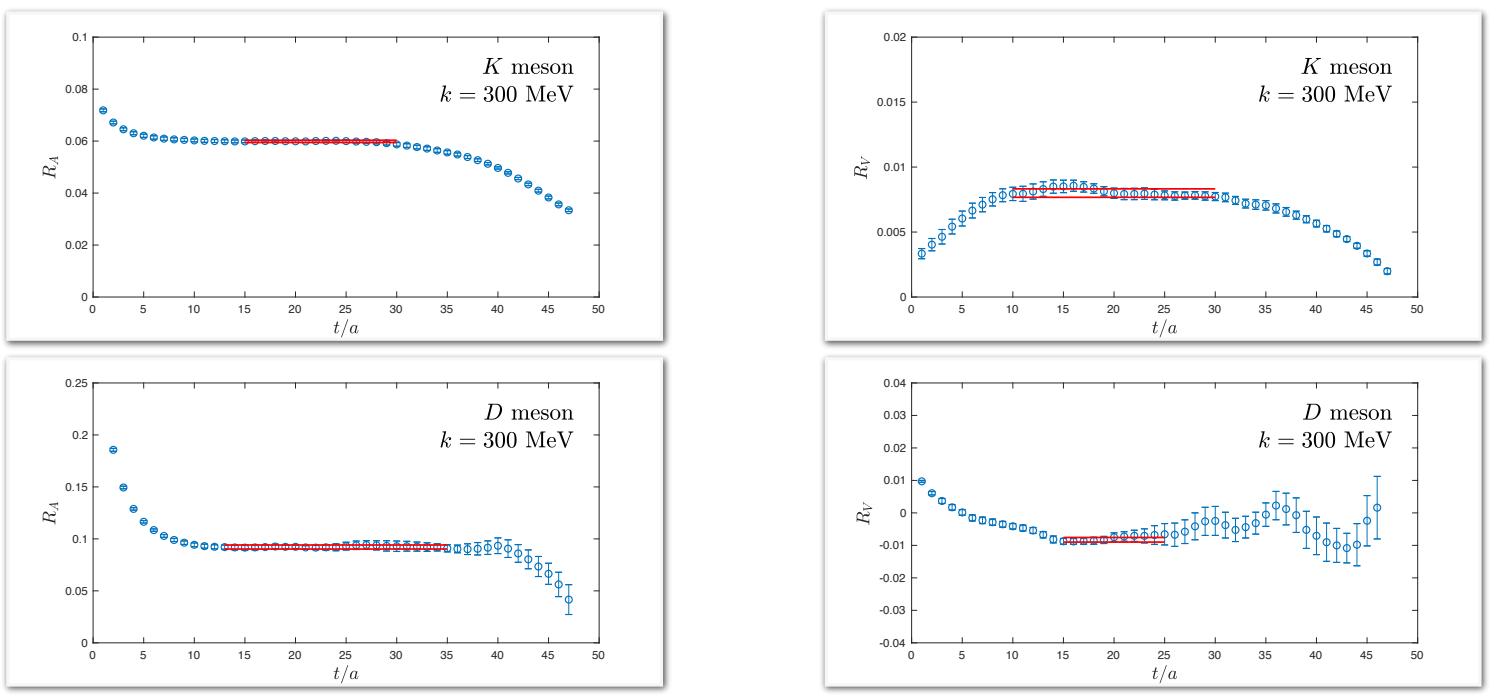

Figure 2: Examples of plateaux fits for the ratios $R_{A}(t, T / 2)$ (left) and $R_{V}(t, T / 2)$ (right).

For $t \gg 0$ we get the following numerical estimators for the form-factors

$$
\begin{aligned}
& R_{A}(t)=\frac{m_{P}}{4 p \cdot k} \sum_{r=1,2} \sum_{j=1,2} \frac{R_{A}^{j r}(t ; \boldsymbol{p}, \boldsymbol{k})}{\varepsilon_{r}^{j}} \rightarrow\left[F_{A}\left(x_{\gamma}\right)+\frac{2 f_{P}}{m_{P} x_{\gamma}}\right], \\
& R_{V}(t)=\frac{m_{P}}{4} \sum_{r=1,2} \sum_{j=1,2} \frac{R_{V}^{j r}(t ; \boldsymbol{p}, \boldsymbol{k})}{i\left(E_{\gamma} \boldsymbol{\varepsilon}_{r} \wedge \boldsymbol{p}-E \boldsymbol{\varepsilon}_{r} \wedge \boldsymbol{k}\right)^{j}} \rightarrow F_{V}\left(x_{\gamma}\right) .
\end{aligned}
$$

At finite $T$, by using the formulae above which are valid for $t>0$, we fit the ratios $R_{A, V}(t)$ by searching a plateau in the region $0 \ll t \ll T / 2$. We also exploit time-reversal symmetries to include the plateaus of $R_{A, V}(t)$ obtained at $t>T / 2$. The values of the meson energies and of the matrix element $\langle P(\boldsymbol{p})|P| 0\rangle$ needed to build these estimators are obtained from standard effectivemass/residue analyses of pseudoscalar-pseudoscalar two-point functions. The pseudoscalar-axial two-point function is used to extract the decay constants $f_{P}$ in order to separate $F_{A}$ from the pointlike contribution $2 f_{P} /\left(m_{P} x_{\gamma}\right)$.

\section{Numerical results}

All the results presented in this section are preliminary. We have used the gauge configurations given in table II of ref. [13], produced with $2+1+1$ twisted mass fermions at three different values of the lattice spacing, $a[\mathrm{fm}]=0.0885(36), 0.00815(30), 0.0619(18)$, with meson masses in the range $250-1930 \mathrm{MeV}$. In total we have included 100 different combinations of momenta obtained by assigning to each of the $\theta_{i=0, t, s}$ five different values; making the same assignements for all choices of the quark masses. All the plots below correspond to the case of $K$ and $D_{(s)}$ mesons at unphysical values of the $\overline{\mathrm{MS}}$ renormalised light-quark mass, $m_{u d}(2 \mathrm{GeV})=11.7 \mathrm{MeV}$, and have been obtained from a simulation at $a=0.0619 \mathrm{fm}$. Thus the reference meson masses are 

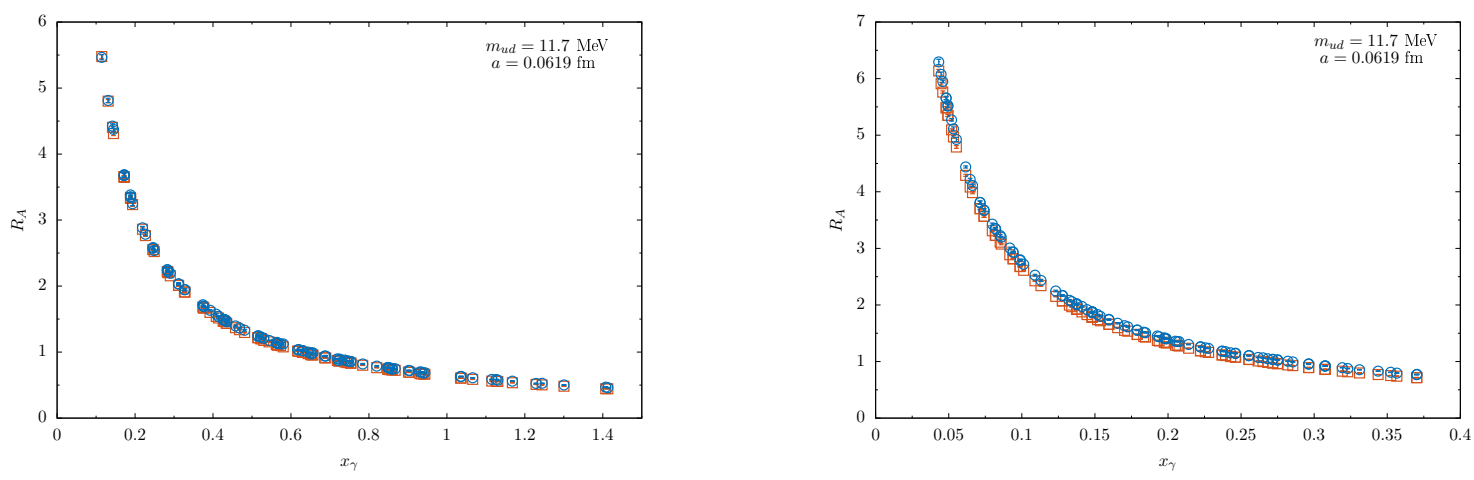

Figure 3: The extracted value of $R_{A}\left(x_{\gamma}\right)$, Eq. (3.5), as a function of $x_{\gamma}$ for the $K$ meson (left) and for the $D_{s}$ meson (right). The (red) squares represent the point-like contribution given by $2 f_{P} /\left(m_{P} x_{\gamma}\right)$.
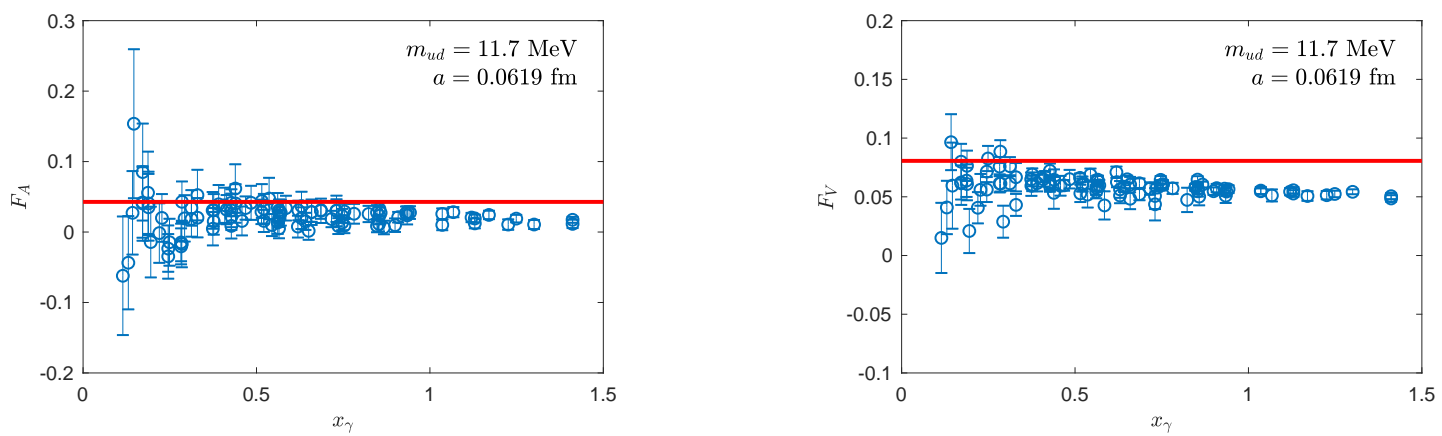

Figure 4: The extracted value of the kaon form factors $F_{A}\left(x_{\gamma}\right)$ (left) and $F_{V}\left(x_{\gamma}\right)$ (right) as a function of $x_{\gamma}$. The (red) lines correspond to the $\chi P T$ predictions obtained by using the formulae discussed in the text.

$M_{D}=1933(50) \mathrm{MeV}, M_{K}=535(14) \mathrm{MeV}$ and $M_{\pi}=255(7) \mathrm{MeV}$. Similar plots can be shown for other values of the simulated parameters. In Fig. 2 we show examples of plateaux for the ratios $R_{A, V}(t)$ for the $K$ and $D$ mesons. This figure is representative of the signal quality, also for other values of masses and momenta. In Fig. 3 we show the extracted value of $R_{A}\left(x_{\gamma}\right)$, Eq. (3.4), for the $K$ meson and for the $D_{s}$ meson. In both cases the point-like contribution, corresponding to the term $2 f_{P} /\left(m_{P} x_{\gamma}\right)$ dominates the form factor. From the measured decay constant and mass, we can subtract the point-like term and extract $F_{A}\left(x_{\gamma}\right)$. In the left-hand plot of Fig. 4 we show $F_{A}\left(x_{\gamma}\right)$ as a function of $x_{\gamma}$ and compare it to the lowest non-trivial order in chiral perturbation theory $\chi P T$, given by $F_{A}\left(x_{\gamma}\right)=$ const. $=8 m_{K}\left(L_{9}^{r}+L_{10}^{r}\right) / f_{K}$, indicated by a line with $L_{9}^{r}+L_{10}^{r} \simeq 0.0017$ [18]. On the right hand plot of Fig. 4 we can compare the directly computed value of $F_{V}$ to its $\chi P T$ prediction, $F_{V}\left(x_{\gamma}\right)=$ const. $=m_{K} /\left(4 \pi^{2} f_{K}\right)$. In a first exploratory study we covered the full physical range of $x_{\gamma}$ in the kaon case (indeed we even have data for unphysical values corresponding to $x_{\gamma}>1$ ) and for $0 \leq x_{\gamma} \leq 0.4$, corresponding to $E_{\gamma} \lesssim 400 \mathrm{MeV}$, for the $D_{s}$ meson. We are currently improving our lattice data and, after a detailed analysis of all the systematics, we shall provide first-principles phenomenologically relevant results for the form factors in the full kinematical range for both light and heavy mesons. The form factors for heavy mesons will represent in this respect a totally 
unexplored field of investigation while, in the case of light mesons, our first-principle results will make it possible to avoid $\chi P T$ in phenomenological analyses.

In conclusion we have shown that, with moderate statistics, it is possible to extract with good precision the form factors relevant for $P \rightarrow \ell \bar{v}_{\ell} \gamma$ decays for both light and heavy mesons and that it is possible to study their momentum dependence. In the near future we will be able to compare the precise theoretical predictions with experimental measurements.

\section{Acknowledgments}

We acknowledge use of CPU time on Marconi-KNL at CINECA within the PRACE project Pra17-4394. V.L., G.M. and S.S. thank MIUR (Italy) for partial support under the contract PRIN 2015. C.T.S. was supported by an Emeritus Fellowship from the Leverhulme Trust. N.T. acknowledges the University of Rome Tor Vergata for the support granted to the project PLNUGAMMA.

\section{References}

[1] M. Tanabashi et al. [Particle Data Group], Phys. Rev. D 98 (2018) no.3, 030001.

[2] C. Kane, C. Lehner, S. Meinel and A. Soni, arXiv:1907.00279 [hep-lat].

[3] J. Bijnens and P. Talavera, Nucl. Phys. B 489 (1997) 387 [hep-ph/9610269].

[4] C. Q. Geng, I. L. Ho and T. H. Wu, Nucl. Phys. B 684 (2004) 281 [hep-ph/0306165].

[5] V. Mateu and J. Portoles, Eur. Phys. J. C 52 (2007) 325 [arXiv:0706.1039 [hep-ph]].

[6] R. Unterdorfer and H. Pichl, Eur. Phys. J. C 55 (2008) 273 [arXiv:0801.2482 [hep-ph]].

[7] V. Cirigliano, G. Ecker, H. Neufeld, A. Pich and J. Portoles, Rev. Mod. Phys. 84 (2012) 399 [arXiv:1107.6001 [hep-ph]].

[8] N. Carrasco et al., Phys. Rev. D 91 (2015) no.7, 074506 [arXiv:1502.00257 [hep-lat]].

[9] V. Lubicz et al. , Phys. Rev. D 95 (2017) no.3, 034504 [arXiv:1611.08497 [hep-lat]].

[10] V. Lubicz et al., PoS LATTICE 2016 (2016) 290 [arXiv:1610.09668 [hep-lat]].

[11] N. Tantalo et al., arXiv:1612.00199 [hep-lat].

[12] D. Giusti et al., Phys. Rev. Lett. 120 (2018) no.7, 072001 [arXiv:1711.06537 [hep-lat]].

[13] M. Di Carlo et al., Phys. Rev. D 100 (2019) no.3, 034514 [arXiv:1904.08731 [hep-lat]]

[14] D. Becirevic, B. Haas and E. Kou, Phys. Lett. B 681 (2009) 257 [arXiv:0907.1845 [hep-ph]].

[15] J. Bijnens, G. Ecker and J. Gasser, Nucl. Phys. B 396 (1993) 81 [hep-ph/9209261].

[16] G. M. de Divitiis, R. Petronzio and N. Tantalo, Phys. Lett. B 595 (2004) 408 [hep-lat/0405002].

[17] C. T. Sachrajda and G. Villadoro, Phys. Lett. B 609 (2005) 73 [hep-lat/0411033].

[18] J. Bijnens and G. Ecker, Ann. Rev. Nucl. Part. Sci. 64 (2014) 149 [arXiv:1405.6488 [hep-ph]]. 